\title{
$H$-SPACES AND THE SUSPENSION HOMOMORPHISM
}

\author{
H. B. HASLAM ${ }^{1,2}$
}

\begin{abstract}
If a space $X$ is an $H$-space, then the homotopy suspension homomorphism is a monomorphism onto a direct factor in all dimensions. We present an example to show that the converse is
\end{abstract} false.

1. Introduction. Let $X$ be an $H$-space and a connected $\mathrm{CW}$ complex. Then the inclusion map $\iota: X \rightarrow \Omega \Sigma X$, defined by $\iota(x)(t)$ $=(x, t) \in \Sigma X$, has a left homotopy inverse. (This was first proved for countable CW-complexes by I. M. James in [6]. For a semisimplicial argument which removes the hypothesis of countability see $[4, \mathrm{p}$. 208] or, for a geometric argument, see [3].) It follows that the suspension homomorphism $\iota *: \pi_{m}(X) \rightarrow \pi_{m}(\Omega \Sigma X)$ is a monomorphism onto a direct factor for all $m$. We call a space $Y$ which satisfies this algebraic criterion $\left(\iota_{*}\right.$ is a monomorphism onto a direct factor in all dimensions) a $\Sigma$-space and we call $Y$ a $\sigma$-space if $\iota *$ is a monomorphism in all dimensions. The purpose of this note is to exhibit a $\Sigma$-space which is not an $H$-space.

As one would expect, the example we have in mind is an infinite $\mathrm{CW}$-complex and we ask whether or not there are finite CW-complexes which are $\Sigma$-spaces and not $H$-spaces. This question is similar to one raised by G. T. Porter: A $\Sigma$-space (in fact a $\sigma$-space) has the property that its higher order spherical Whitehead products (HOWP) vanish [8, Corollary 4]. In [9], Porter asked whether there are finite $\mathrm{CW}$-complexes having trivial HOWP which are not $H$ spaces; to our knowledge, Porter's question is still unresolved.

In what follows we will denote inclusion maps by $\iota, \iota^{\prime}$ etc. and Hurewicz homomorphisms by $h, h^{\prime}$ etc.

2. The example. Consider the two-stage Postnikov system

$$
K\left(Z_{2}, 2 n-1\right) \rightarrow E_{n} \rightarrow K(Z, 2) \quad(n \geqq 2)
$$

with $k$-invariant $\alpha^{n}$, where $\alpha$ is the generator of $H^{2}\left(Z, 2 ; Z_{2}\right)$.

Received by the editors November 24, 1969.

AMS 1969 subject classifications. Primary 5540.

Key words and phrases. $H$-space, suspension homomorphism.

1 This work was supported in part by the National Science Foundation under Grant NSF GP-7913 and in part by the Graduate Division of the University of California at Irvine.

2 This work constituted a portion of the author's dissertation which was written under the direction of Professor George S. McCarty. 
Theorem 1. (1) $E_{3}$ is a $\Sigma$-space.

(2) $E_{n}$ is a $\sigma$-space for all $n$.

(3) $E_{n}$ is an H-space if and only if $n=2^{k}$.

Part (3) of Theorem 1 follows from the fact that the $k$-invariant $\alpha^{n}$ is primitive if and only if $n=2^{k}$ [7]. As a first step to proving parts (1) and (2) of Theorem 1, we replace $E_{n}$ by an equivalent CW-complex.

Let $C P^{n-1}$ denote the $(n-1)$-dimensional complex projective space and $p_{n-1}: S^{2 n-1} \rightarrow C P^{n-1}$ the usual fibration with fiber $S^{1}$. Then $C P^{n}=C P^{n-1} \cup_{p_{n-1}} e^{2 n}$. Starting with $C P^{1}=S^{2}$ and the Hopf map $p_{1}: S^{3} \rightarrow S^{2}$ we see that $C P^{n}$ has a $C W$-structure with exactly one cell in each even dimension $\leqq 2 n$. Let $d_{n-1}: S^{2 n-1} \rightarrow S^{2 n-1}$ be a map of degree 2 and set $X_{n}=C P^{n-1} \bigcup_{p_{n-1} d_{n-1}} e^{2 n}$. Let $Y_{n}$ be the space obtained from $X_{n}$ by attaching $m$-cells, $m \geqq 2 n+1$, so as to "kill" its homotopy groups in dimensions $\geqq 2 n$.

Lemma 1. The spaces $Y_{n}$ and $E_{n}$ have the same homotopy type.

Proof. Since $p_{n-1} d_{n-1}$ represents $\pm 2 \in Z=\pi_{2 n-1}\left(C P^{n-1}\right)$, it is clear that $Y_{n}$ and $E_{n}$ have the same homotopy groups and so $Y_{n}$ also has a Postnikov system of the form

$$
K\left(Z_{2}, 2 n-1\right) \rightarrow E_{n}^{\prime} \rightarrow K(Z, 2) .
$$

Furthermore, there is a map $Y_{n} \rightarrow E_{n}^{\prime}$ which is a homotopy equivalence. Since $H^{2 n}\left(Z, 2 ; Z_{2}\right)=Z_{2}$, the $k$-invariant of this Postnikov system is either 0 or $\alpha^{n}$. If the $k$-invariant were 0 , we would have $E_{n}^{\prime}=K(Z, 2) \times K\left(Z_{2}, 2 n-1\right)$, but then $H^{2 n-1}\left(E_{n}^{\prime} ; Z_{2}\right)=Z_{2}$ whereas $H^{2 n-1}\left(Y_{n} ; Z_{2}\right)=0$, since $Y_{n}$ has no $(2 n-1)$-cells. We conclude that the $k$-invariant is $\alpha^{n}$ and that $E_{n}^{\prime}=E_{n}$ which establishes the lemma.

LeMma 2. $\iota_{*}: \pi_{2}\left(Y_{n}\right) \rightarrow \pi_{2}\left(\Omega \Sigma Y_{n}\right)$ is an isomorphism.

PROof. This follows from the homotopy suspension theorem.

It remains to consider $\iota_{*}: \pi_{2 n-1}\left(Y_{n}\right) \rightarrow \pi_{2 n-1}\left(\Omega \Sigma Y_{n}\right)$ or equivalently, by a cellular approximation argument, $\iota_{*}: \pi_{2 n-1}\left(X_{n}\right) \rightarrow \pi_{2 n-1}\left(\Omega \Sigma X_{n}\right)$.

From the definition of $X_{n}$ and $C P^{n}$ we see that there is a map $f_{n}: X_{n} \rightarrow C P^{n}$ which maps the subspace $C P^{n-1}$ of $X_{n}$ and $C P^{n}$ identically. In the following we will consider $f_{n}$ to be an inclusion and, by abuse of notation, will consider the "pair" $\left(C P^{n}, X_{n}\right)$. Set $A_{n}=\Omega \Sigma X_{n}$, $B_{n}=\Omega \Sigma C P^{n}$ and $g_{n}=\Omega \Sigma f_{n}: A_{n} \rightarrow B_{n}$.

LEMMA 3. In the following diagram all the homomorphisms are isomorphisms and the groups are isomorphic to $Z_{2}$. 


$$
\begin{array}{ccc}
\pi_{2 n}\left(C P^{n}, X_{n}\right) & \rightarrow \pi_{2 n}\left(B_{n}, A_{n}\right) \\
h_{1} \downarrow & \downarrow h_{2} \\
H_{2 n}\left(C P^{n}, X_{n}\right) \stackrel{\iota *}{\rightarrow} H_{2 n}\left(B_{n}, A_{n}\right)
\end{array}
$$

Proof. Clearly

$$
\begin{aligned}
H_{m}\left(X_{n}\right)=H_{m}\left(C P^{n}\right) & =Z & & m=0,2, \cdots, 2 n, \\
& =0 & & \text { otherwise. }
\end{aligned}
$$

Moreover, we may choose generators $a_{2 m}$ and $b_{2 m}$ of $H_{2 m}\left(X_{n}\right)$ and $H_{2 m}\left(C P^{n}\right)$, respectively, so that

$$
\begin{array}{rlrl}
f_{n^{*}}\left(a_{2 m}\right) & =b_{2 m} & & 0 \leqq m \leqq n-1, \\
& =2 \cdot b_{2 n} & m & =n .
\end{array}
$$

Consequently, the pair $\left(C P^{n}, X_{n}\right)$ is $(2 n-1)$-connected; by the Hurewicz theorem $h_{1}$ is an isomorphism. Plainly $H_{2 n}\left(C P^{n}, X_{n}\right)=Z_{2}$.

The Pontrjagin ring $H_{*}\left(A_{n}\right)\left(H_{*}\left(B_{n}\right)\right)$ is the free associative algebra on $n$ generators, namely $\alpha_{2}, \cdots, \alpha_{2 n}$ (resp. $\beta_{2}, \cdots, \beta_{2 n}$ ), where $\alpha_{2 m}=\iota_{*}\left(a_{2 m}\right)$ and $\beta_{2 m}=\iota_{*}\left(b_{2 m}\right), m=1, \cdots, n[1]$. Since the map $g_{n}$ is an $H$-map, it follows that the induced homomorphism $g_{n^{*}}: H_{m}\left(A_{n}\right)$ $\rightarrow H_{m}\left(B_{n}\right)$ is an isomorphism for $m \leqq 2 n-1$ and has cokernel $Z_{2}$ in dimension $2 n$. As above we conclude that $h_{2}$ is an isomorphism and that $H_{2 n}\left(B_{n}, A_{n}\right)=Z_{2}$. Finally, the homomorphism $\iota_{*}$ is easily seen to be an epimorphism and therefore an isomorphism. This completes the proof of the lemma.

Consider Figure 1. That the leftmost vertical homomorphism

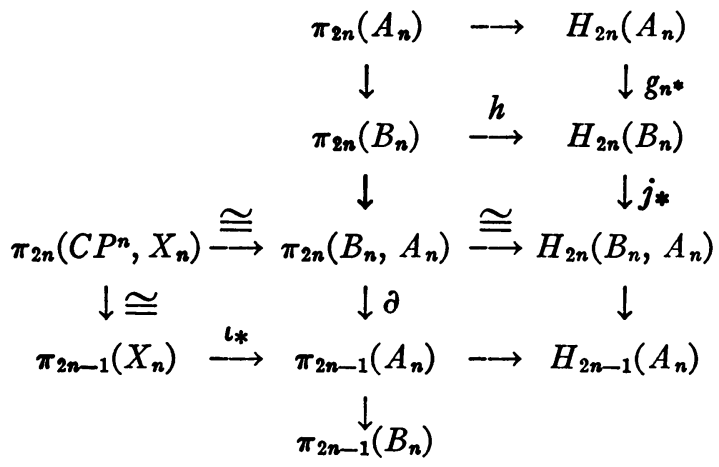

FIGURE 1

is an isomorphism follows from the fact that $\pi_{2 n}\left(C P^{n}\right)=\pi_{2 n-1}\left(C P^{n}\right)=0$ and by Lemma 3 the homomorphisms in the third row are isomor- 
phisms. It follows that $\iota_{*}$ is a monomorphism if and only if $j_{*} h=0$ and that $\iota_{*}$ is an epimorphism if and only if $\pi_{2 n-1}\left(B_{n}\right)=\pi_{2 n}\left(\Sigma C P^{n}\right)=0$.

Proof of PART (2) OF Theorem 1. By the foregoing remark it suffices to show that $j_{*} h=0$. Now any element of $H_{2 n}\left(B_{n}\right)$ can be expressed as $r \cdot \beta_{2 n}+g_{n^{*}}(y), r=0,1, y \in H_{2 n}\left(A_{n}\right)$ (see the proof of Lemma 3 ). To show $j_{*} h=0$, it suffices to show that no element of the form $\beta_{2 n}+g_{n^{*}}(y)$ is in the image of $h$. Let $x \in \pi_{2 n}\left(B_{n}\right)$ and, in order to obtain a contradiction, assume that $h(x)=\beta_{2 n}+g_{n^{*}}(y)$. Write $y=s \cdot \alpha_{2 n}+z$, where $z$ is in the subalgebra of $H_{*}\left(A_{n}\right)$ generated by the set $\left\{\alpha_{2}, \cdots, \alpha_{2 n-2}\right\}$ and $s$ is some integer. Consider Figure 2,

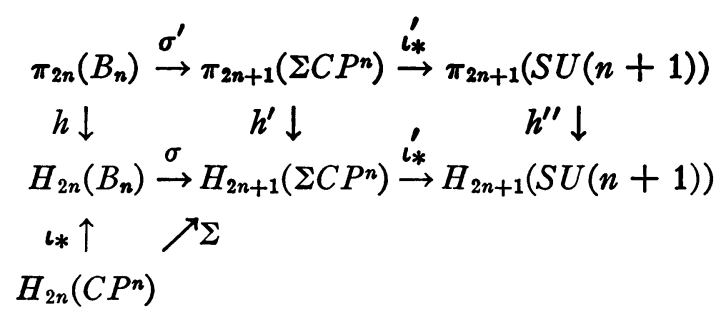

FigURE 2

where $\sigma$ denotes the homology suspension, $\sigma^{\prime}$ denotes the usual bijection and $\iota^{\prime}$ denotes the inclusion map. (The special unitary group $S U(n+1)$ can be given a $C W$-structure with $\Sigma C P^{n}$ as a subcomplex.) From the (signed) commutativity of Figure 2 and the relations $g_{n^{*}}\left(\alpha_{2 n}\right)=2 \cdot \beta_{2 n}$ and $\sigma\left(g_{n^{*}}(z)\right)=0[11$, Corollary 6.2] we see that $h^{\prime \prime} \iota_{*}^{\prime} \sigma^{\prime}(x)=(2 s+1) \cdot \iota_{*}^{\prime} \Sigma\left(b_{2 n}\right)$. Toda [10] has shown that there is an element $\zeta \in \pi_{2 n+1}\left(\Sigma C P^{n}\right)$ such that $\iota_{*}^{\prime}(\zeta)$ generates $\pi_{2 n+1}(S U(n+1))=Z$ and $h^{\prime}(\zeta)=n ! \cdot \Sigma\left(b_{2 n}\right)$. Therefore, there is an integer $t$ such that $\iota_{*}^{\prime} \sigma^{\prime}(x)=t \cdot \iota_{*}^{\prime}(\zeta)$ and we compute $h^{\prime \prime} \iota_{*}^{\prime} \sigma^{\prime}(x)=t \cdot n ! \cdot \iota_{*}^{\prime} \Sigma\left(b_{2 n}\right)$. Since $\iota_{*}^{\prime} \Sigma\left(b_{2 n}\right)$ is of infinite order in $H_{2 n+1}(S U(n+1))$ we cannot have an even multiple of this element equal to an odd multiple. This gives the desired contradiction and completes the proof of part (2) of Theorem 1.

LEMMA 4. $\pi_{2 n}\left(\Sigma C P^{n}\right)=0$ if and only if $\pi_{2 n}\left(\Sigma C P^{n-1}\right)=Z_{n !}$.

Proof. Consider the following exact sequence due to J. H. C. Whitehead [5, p. 115]

$$
\begin{aligned}
\rightarrow \pi_{2 n+1}\left(\Sigma C P^{n}\right) & \stackrel{h^{\prime}}{\rightarrow} H_{2 n+1}\left(\Sigma C P^{n}\right) \rightarrow \Gamma_{2 n}\left(\Sigma C P^{n}\right) \\
& \rightarrow \pi_{2 n}\left(\Sigma C P^{n}\right) \rightarrow H_{2 n}\left(\Sigma C P^{n}\right) \rightarrow .
\end{aligned}
$$


It follows from the definition of $\Gamma_{2 n}$ that $\Gamma_{2 n}=\pi_{2 n}\left(\Sigma C P^{n-1}\right)$. Since $H_{2 n}\left(\Sigma C P^{n}\right)=0$, the lemma will follow if we show that $\operatorname{Im}\left(h^{\prime}\right)=n$ ! $\cdot Z \subset Z=H_{2 n+1}\left(\Sigma C P^{n}\right)$. But this follows from the result of Toda cited above and the fact that $h^{\prime \prime}$ (see Figure 2) is a monomorphism [2].

Proof of PART (1) OF Theorem 1. As noted above, it suffices to show that $\pi_{6}\left(\Sigma C P^{3}\right)=0$ or, by Lemma 4 , that $\pi_{6}\left(\Sigma C P^{2}\right)=Z_{6}$. One can compute $\pi_{6}\left(\Sigma C P^{2}\right)=Z_{6}$ by considering the homotopy sequence of the pair $\left(\Sigma C P^{2}, \Sigma S^{2}\right)$ and making use of the fact that $Z_{6}$ is a subgroup of $\pi_{6}\left(\Sigma C P^{2}\right)$ (see the proof of Lemma 4 ).

In conclusion we point out a contrast between the Hurewicz homomorphism and the suspension homomorphism. We make no attempt to be precise here; precise statements are given in [3].

Let $X^{\infty}$ denote the infinite symmetric product space of $X$ and let $\iota: X \rightarrow X^{\infty}$ be the inclusion map. As is well known, the geometric condition that $X$ be dominated by $X^{\infty}$ is equivalent to the associated algebraic condition that the induced homomorphism $\iota_{*}: \pi_{m}(X)$ $\rightarrow \pi_{m}\left(X^{\infty}\right) \cong H_{m}(X)$ (i.e. the Hurewicz homomorphism) be a monomorphism onto a direct factor in all dimensions.

Now let $X_{\infty}$ denote the reduced product space of $X$ and let $\iota: X \rightarrow X_{\infty}$ be the inclusion map. Our example shows that the geometric condition that $X$ be dominated by $X_{\infty}$ (i.e. that $X$ be an $H$-space) is not equivalent to the associated algebraic condition that the induced homomorphism $\iota *: \pi_{m}(X) \rightarrow \pi_{m}\left(X_{\infty}\right) \cong \pi_{m+1}(\Sigma X)$ (i.e. the suspension homomorphism) be a monomorphism onto a direct factor in all dimensions.

\section{BIBLIOGRAPHY}

1. R. Bott and H. Samelson, On the Pontryagin product in spaces of paths, Comment. Math. Helv. 27 (1953), 320-337. MR 15, 643.

2. H. Cartan and J. P. Serre, Espaces fibrés et groupes d'homotopie. II: Applications, C. R. Acad. Sci. Paris 234 (1952), 393-395. MR 13, 675.

3. H. B. Haslam, G-spaces and H-spaces, Thesis, University of California at Irvine, 1969.

4. Peter Hilton, Homotopy theory and duality, Gordon and Breach, New York, 1965. MR $33 \# 6624$.

5. - An introduction to homotopy theory, Cambridge Tracts in Math. and Math. Phys., no. 43, Cambridge Univ. Press, New York, 1953. MR 15, 52.

6. I. M. James, Reduced product spaces, Ann. of Math. (2) 62 (1955), 170-197. MR 17, 396.

7. Donald W. Kahn, Induced maps for Postnikov systems, Trans. Amer. Math. Soc. 107 (1963), 432-450. MR $27 \# 764$.

8. Gerald J. Porter, Higher-order Whitehead products, Topology 3 (1965), 123-135. MR 30 \#4261. 
9. - Spaces with vanishing Whitehead products, Quart. J. Math. Oxford Ser. (2) 16 (1965), 77-84. MR 30 \#2511.

10. Hirosi Toda, $A$ topological proof of the theorems of Bott and Borel-Hirzebruch for homotopy groups of unitary groups, Mem. Coll. Sci. Univ. Kyoto Ser. A Math. 32 (1959), 103-119. MR 21 \#7502.

11. George W. Whitehead, On the homology suspension, Ann. of Math. (2) 62 (1955), 254-268. MR 17, 520.

Florida State University, Tallahassee, Florida 32306 